\title{
The Evolution of the Baryonic Tully-Fisher Relation over the past 6 Gyr
}

\author{
M. Puech ${ }^{1}$, F. Hammer ${ }^{1}$, H. Flores ${ }^{1}$, R. Delgado-Serrano ${ }^{1,2}$, \\ M. Rodrigues ${ }^{1,4,5}$, and Y. Yang ${ }^{1,5}$ \\ ${ }^{1}$ GEPI, Observatoire de Paris, CNRS, University Paris Diderot; 5 Place Jules Janssen, 92190 \\ Meudon, France \\ email: mathieu.puech@obspm.fr \\ ${ }^{2}$ Panama Observatory, Technological University of Panama, 0819-07289 Panama, Rep. of \\ Panama \\ ${ }^{4}$ CENTRA, Instituto Superior Tecnico, Av. Rovisco Pais 1049-001 Lisboa, Portugal \\ ${ }^{5}$ European Southern Observatory, Casilla 19001, Santiago 19, Chile \\ ${ }^{6}$ National Astronomical Observatories, Chinese Academy of Sciences, 20A Datun Road, \\ Chaoyang District, Beijing 100012, China
}

\begin{abstract}
Scaling relations are salient ingredients of galaxy evolution and formation models. I summarize results from the IMAGES survey, which combines spatially-resolved kinematics from FLAMES/GIRAFFE with imaging from HST/ACS and other facilities. Specifically, I will focus on the evolution of the stellar mass and baryonic Tully-Fisher Relations (TFR) from $\mathrm{z}=0.6$ down to $\mathrm{z}=0$. We found a significant evolution in zero point and scatter of the stellar mass TFR compared to the local Universe. Combined with gas fractions derived by inverting the Schmidt-Kennicutt relation, we derived for the first time a baryonic TFR at high redshift. Conversely to the stellar mass TFR, the baryonic relation does not appear to evolve in zero point, which suggests that most of the reservoir of gas converted into stars over the past $6 \mathrm{Gyr}$ was already gravitationally bound to galaxies at $\mathrm{z}=0.6$.
\end{abstract}

Keywords. galaxies: evolution, galaxies: high-redshift, galaxies: interactions, galaxies: kinematics and dynamics

\section{Introduction}

The distant Tully-Fisher Relation (TFR) has been extensively studied over the past two decades. One of its most intriguing property is that it shows a significantly larger scatter at high redshift compared to the local Universe. This effect is seen both in B-band and in K-band (e.g., Böhm \& Ziegler 2007, Conselice et al. 2005), which indicates that it is not related to dust or stellar population effects.

Integral Field Spectroscopy (IFS) now allows us to study the spatially-resolved kinematics of distant galaxies. First studies of the distant TFR using IFS revealed a correlation between the scatter of the K-band TFR at $\mathrm{z} \sim 0.6$ and the dynamical state of galaxies, meaning that the larger the scatter in the TFR, the larger the dynamical disturbances observed in the galaxy kinematics (Flores et al. 2006). It was shown that once reduced to rotating disks, the $\mathrm{z} \sim 0.6 \mathrm{~K}$-band TFR has the same scatter that the local relation, which indicates that the scatter in the distant TFR is indeed entirely due to kinematic disturbances, strong enough to destroy rotation on large spatial scales. This led Puech et al. (2008) to suggest that major mergers are the physical driver for such kinematic disturbances in a large fraction of $\mathrm{z} \sim 0.6$ galaxies (see also Neichel et al. 2008 from a morphological point of view). 
Interestingly, a new kinematic tracer called $S$ was proposed by Weiner et al. (2006). This tracer combines both the rotation velocity and the velocity dispersion, which makes it a tracer of the total kinematic energy. Kassin et al. (2007) showed that, when applied to the TFR, one gets a new relation (hereafter $S$-TFR), on which the scatter is significantly reduced compared to the "regular" TFR (i.e., when the rotation velocity only is considered). Covington et al. (2009) used hydrodynamical simulations to suggest that such an effect on the scatter could result from shocks in the gaseous phase during galaxy major mergers. These shocks would lead to a transfer of energy from bulk motions (i.e., large-scale rotation) to random motions, ocuring at smaller spatial scales. This transfer of energy translates into a drop in the rotation velocity as a function of time while the velocity dispersion increases, which results in $S$ staying constant at first order. This would explain why the $S$-TFR shows a much reduced scatter compared to the regular TFR.

\section{The IMAGES stellar-mass (smTFR) and $S$-TFR}

We studied both the stellar-mass (smTFR) and $S$-TFR in the IMAGES sample. The IMAGES sample is a representative sample of $63 \mathrm{M}^{*}$ emission line galaxies selected in four different fields of view (i.e., CFRS03, CFRS22, HDFS, and CDFS, see Yang et al. 2008). All galaxies were observed using the FLAMES/GIRAFFE IFUs, which allowed us to map their kinematics at relatively large spatial scales $(\sim 3.5 \mathrm{kpc} / \mathrm{pix}$ at $\mathrm{z} \sim 0.6)$. We derived the rotation velocities using Monte-Carlo simulations, taking carefully into account beam smearing effects (Puech et al. 2008). Stellar masses were obtained from (dust and inclination corrected) K-band luminosities and B-V colors using the Bell et al. (2003) simplified prescriptions. We assumed a diet Salpeter IMF, which, in combination to the Bell et al. (2003) prescriptions for stellar masses, has the interesting property of maximizing the stellar mass estimates, meaning that any other combination of stellar population model and IMF would lead to smaller masses (Hammer et al. 2009). This means that any shift obtained with this combination between the distant and local relation would be larger with any other combination.

The derived smTFR at $\mathrm{z} \sim 0.6$ is shown on the left panel of Fig. 1. It confirms the results of Puech et al. (2008), that the entire scatter of the distant relation is due to dynamically non-relaxed galaxies. Once reduced to rotating disks, the smTFR is shifted downward by $0.34_{-0.06}^{0.21}$ dex (where the quoted errobars represent systematic uncertainties) compared to the local relation, which suggests that intermediate-mass, emission line rotating disks grew up by a factor $\sim 2$ in stellar mass over the past 6 Gyr. This shift is robust against both random and systematic uncertainties (see Puech et al. 2010 for details).

Similar results were obtained at $\mathrm{z} \sim 2.2$ in the SINS survey: Cresci et al. (2009) found a $\mathrm{z} \sim 2.2$ relation shifted downward by 0.41 dex compared to the location relation. At first sight, it might however be surprising to find a similar shift at $\mathrm{z} \sim 0.6$ and $\mathrm{z} \sim 2$ compared to the local relation. We showed in Puech et al. (2010) that this is linked to the local sample used to derive the shift in zero point. Several widely used local comparison samples were indeed compared in Hammer et al. (2007), where it was shown that the Verheijen (2001) Ursa Major sample is biased towards low-mass galaxies, when compared to the local velocity function. Other samples, e.g., the Courteau (1997) sample drawn from the UGC catalog or the Pizagno et al. (2007) drawn from the SDSS, appear to more appropriate when one compares representative samples at different redshifts. In the IMAGES survey, we used the SDSS sample as a reference, while the SINS survey relied on the Bell \& de Jong (2001) local smTFR, which was drawn from the Verheijen (2001) sample. If one re-derives the shift between the $\mathrm{z} \sim 2.2$ smTFR and the SDSS smTFR relation, the 
resulting offset increases from $\sim 0.4$ to $\sim 0.6$ dex, i.e., significantly larger than the shift of 0.36 dex found between the $\mathrm{z} \sim 0.6 \mathrm{smTFR}$ and the local relation. When comparing the TFR between different redhifts, it is therefore very important to adopt representative samples, or at least samples which share the same properties in terms of mass and/or velocity distribution.

We also derived the $S$-TFR in the IMAGES sample (see middle panel of Fig. 1), and confirms the results of Kassin et al. (2007), i.e., the scatter is reduced by a factor $\sim 2$ compared to the regular smTFR. We also found observational evidence for the transfer of energy suggest by Covington et al. (2009). One of the object in the IMAGES sample was indeed found to be characteristic of the shrinking effect in the scatter due to the use of $S$ (see Fig. 1): it was modeled in detail using hydrodynamical simulations by Peirani et al. (2009), who found that the best model for this object corresponds to a 3:1 major merger.
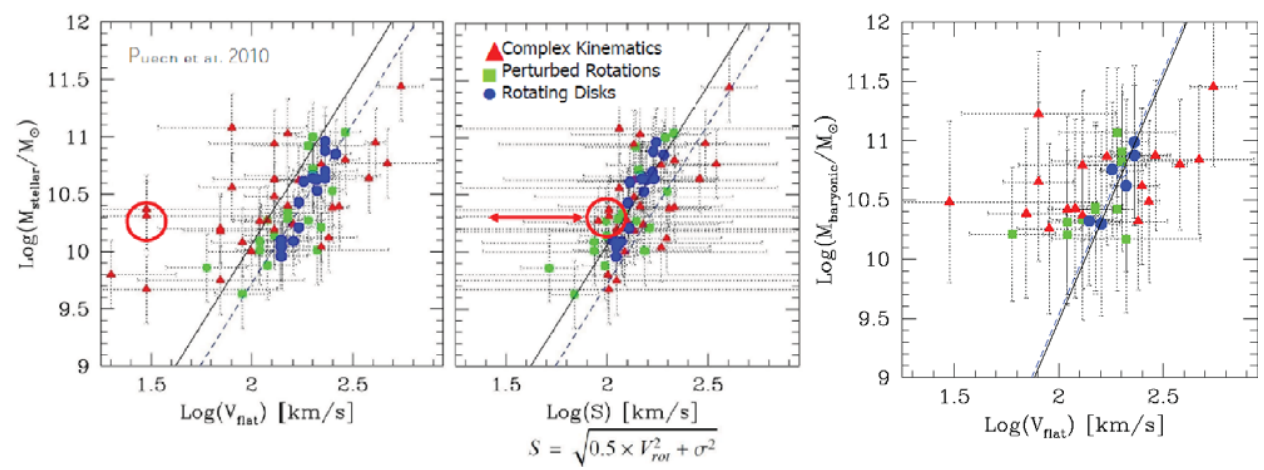

Figure 1. Left: Evolution of the stellar-mass TFR between $\mathrm{z} \sim 0.6$ (dashed line) and $\mathrm{z}=0$ (solid line). Distant rotating disks are shown as dots, perturbed rotators as squares, and galaxies with complex kinematics as triangles. Middle: Same relation but using the kinematic tracer S, which combines rotation velocity with velocity dispersion (see Kassin et al. 2007). The solid and dashed lines are the same that in the left panel. Right: Evolution of the baryonic TFR in the CDFS sub-sample. The solid line is the local bTFR, while the dashed line is a fit to the distant relation. See Puech et al. (2010) for details. The object encircled in red in the two leftest panels correspond to a major merger modeled in detail by Peirani et al. (2009) using hydrodynamical simulations (see text).

\section{The IMAGES baryonic TFR}

We estimate gas masses in the IMAGES sample by inverting the Schmidt-Kennicutt law between star formation rate and gas mass densities Kennicutt (1989). Total SFRs were estimated by summing contributions from the UV and the IR, while total gas radii were estimated using GIRAFFE-IFU [OII] maps carefully deconvolved from beam smearing effects (see Puech et al. 2010). Interestingly, the ionized gas radius is found to be on average $\sim 30 \%$ more extended than the total UV radius, which suggests that another physical process than radiation from OB stars is ionizing gaz at large radii. We found observational evidence that shocks during major mergers could be this alternative driver (Puech et al. 2009). Such a process could also be responsible for the increased velocity dispersion observed in $\mathrm{z} \sim 0.6$ disks compared to local ones (Puech et al. 2007; Epinat et al. 2010), as also observed in higher-redshif disks (Förster Schreiber et al. 2009).

Galaxies at $\mathrm{z} \sim 0.6$ show a median gas fraction of $\sim 31 \pm 1 \%$. The same mean gas fraction was estimated independantly by Rodrigues et al. (2008) relying on the evolution of the 
mass-metallicity relation combined with a closed-box chemical model. This suggests that systematic effects are probably limited and gives us some confidence in the estimated mass of gas in $\mathrm{z} \sim 0.6$ galaxies.

The resulting baryonic TFR (bTFR) is shown on the right panel of Fig. 1. This is the first time that the bTFR is derived at high redshifts. On overall, it shows the same structure that the smTFR. However, conversely to the smTFR that is shifted downward by a factor $\sim 2$ in stellar mass, the bTFR does not show any evidence for evolution in zero point compared to the local relation. This indicates that the reservoir of gas that has been converted into stars over the past 6 Gyr was already gravitationnaly bound to disks at $\mathrm{z} \sim 0.6$. As a consequence, there is no need for external gas accretion, in qualitative agreement with cosmological simulations which predict that cold flows vanish at $\mathrm{z} \leqslant 1.5$ in massive halos where such galaxies inhabit in (Kereš et al. 2009; Puech 2010). This does not mean that there is no gas accretion in these galaxies since within systematic uncertainties, there is room for cold gas accretion at a level of $\sim 30 \%$ of the local baryonic mass, but there is presently no need for such an accretion, at least from a dynamical point of view.

\section{References}

Bell, E. F. \& de Jong, R. S. 2001, ApJ, 550, 212

Bell, E. F., McIntosh, D. H., Katz, N., \& Weinberg, M. D. 2003, ApJS, 149, 289

Böhm, A. \& Ziegler, B. L. 2007, ApJ, 668, 846

Conselice, C. J., Bundy, K., Ellis, R. S., Brichmann, J., Vogt, N. P., \& Phillips, A. C. 2005, ApJ, 628, 160

Courteau, S. 1997, AJ, 114, 2402

Covington, M. D., Kassin, S. A., Dutton, A. A., et al. 2009, ApJ, submitted, astro-ph/0902.0566

Cresci, G., Hicks, E. K. S.., Genzel, R., et al. 2009, ApJ, 697, 115

Epinat, B., Amram, P., Balkowski, C., \& Marcelin, M. MNRAS, 401, 2113

Flores, H., Hammer, F., Puech, M., Amram, P., \& Balkowski, C. 2006, A\&A, 455, 107

Forster Schreiber, N. M., et al. 2009, ApJ, accepted, arXiv:0903.1872

Hammer, F., Puech, M., Chemin, L., Flores, H., \& Lehnert, M. 2007, ApJ, 662, 322

Hammer, F., Flores, H., Puech, M., et al. 2009, A\&A, 507, 1313

Kassin, S. A., Weiner, B. J., Faber, S. M., et al. 2007, ApJ, 660, 35

Kennicutt, R. 1989, ApJ, 344, 685

Kereš, D., Katz, N., Fardal, M., Davé, R., \& Weinberg, D. H. 2009, MNRAS, 395, 160

Neichel, B., et al. 2008, A\& $\mathcal{E}$ A, 484, 159

Peirani, S., Hammer, F., Flores, H., et al. 2009, A\&A, 496, 51

Pizagno, J., Prada, F., Weinberg, D. H., et al. 2007, AJ, 134, 945

Puech, M., Hammer, F., Lehnert, M. D., \& Flores, H. 2007a, A\&A, 466, 83

Puech, M., Flores, H., Hammer, F., et al. 2008, A\& $A$, 484, 173

Puech, M., Hammer, F., Flores, H., Neichel, B., \& Yang, Y. 2009, A\&A, 493, 899

Puech, M., Hammer, F., Flores, H., et al. 2010, A\&SA, 510, 68

Puech, M. 2010, MNRAS, 406, 535

Rodrigues, M., Hammer, F., Flores, H., et al. 2008, A\&SA, 492, 371

Verheijen, M. A. W.. 2001, ApJ, 563, 694

Weiner, B. J., et al. 2006, ApJ, 653, 1027

Yang, Y., Flores, H., Hammer, F., et al. 2008, A\&A, 477, 789 